\title{
Prevalence of Dental Caries and its Association with Risk Factors amongst Preschool Children of Bharakahu, Islamabad
}

\author{
Saba Masoud ${ }^{1}$, Sheze Haroon Qazi ${ }^{2}$, Rubina Mumtaz ${ }^{3}$ \\ ${ }^{1}$ Lecturer, Department of Community Dentistry, Islamabad Medical and Dental College, Pakistan \\ ${ }^{2}$ Assistant Professor, Department of Community Dentistry, Islamabad Medical and Dental College, Pakistan \\ ${ }^{3}$ Associate Professor, Department of Community Dentistry, Islamabad Medical and Dental College, Pakistan
}

\begin{abstract}
A BST RACT
Background: It is necessary to understand the prevalence and pattern of distribution of dental caries for better planning and execution of preventive activities in the right dimension. This study provided the base line data about prevalence of dental caries in pre-school children of Bharakahu, Islamabad, and its association with brushing, sugar consumption and previous dental visits.

Material and Methods: A total of 384 preschool children aged 3-5-year-old were screened from periurban area of Bharakahu, Islamabad, using type IV screening method. Data about decayed, missing and filled teeth was recorded using decayed-missing-filled teeth (DMFT) index. Socio-demographics, brushing, sugar consumption and previous dental visits were also recorded and analyzed and their association with dental caries was assessed using Chi square and Odds ratio.

Results: The overall caries prevalence among the sample was $49 \%$. The mean DMFT score was $2.07 \pm 3.215$. There was no significant difference in caries prevalence in relation to gender of the children. The most significant association was for brushing and low DMFT. Children who brushed their teeth were 4 times more likely to have a low DMFT score of $<1$ than those who did not brush at all $(P<0.001)$. Likewise, those who had visited a dentist in the past were twice more likely to have a low DMFT $(P=0.003)$. The association of low sugar consumption and a low DMFT score was statistically insignificant (OR 1.4; $P=0.878$ ). Hence, brushing turned out to be the most significant factor in determining the presence of caries in a child.

Conclusions: This study provided us with the baseline data regarding the prevalence of caries in primary school going children of Bharakahu. The prevalence of unmet dental treatment needs was reflected through a high number of 'decayed teeth' as compared to missing and filled ones highlighting the need for restorative care in these children.

Key words: Dental caries, Preschool children, Sugar consumption, Tooth brushing

Authors' Contribution:

${ }^{1-3}$ Conception; Literature research; manuscript design and drafting; Critical analysis and manuscript review; Data analysis; Manuscript Editing.

Cite this article. Masoud S, Qazi SH, Mumtaz R. Prevalence of Dental Caries and its Association with Risk Factors amongst Preschool Children of Bharakahu, Islamabad. J
\end{abstract} Islamabad Med Dental Coll.2020; 9(2): 88-94. Doi: 10.35787/jimdc.v9i2.463

\section{Introduction}

Oral health problems in children revolve around dental caries predominantly. ${ }^{1}$ Dental caries is often referred to as pandemic disease, with characteristics of high proportion of untreated caries causing pain, functional restrictions, distress, premature tooth loss, malnutrition and ultimately 
less growth and development. ${ }^{2}$ Functional restrictions also include problems in speech, sleeping, swallowing and breathing etc., all due to pain. $^{3}$ It is necessary for the pediatrician to understand the disease burden (prevalence), pattern of distribution and characteristics. ${ }^{4}$ Dental caries occurs due to association of four factors including susceptible tooth surface, bacteria, duration of exposure to bacteria and diet rich in carbohydrates. $^{3}$

With respect to data collected from 1973 to 2008 on 190 WHO countries, worldwide average DMFT (decayed, missing and filled teeth) index was 2.11 among children. ${ }^{5}$ The prevalence of caries in Asia is estimated to be about $60-90 \%{ }^{6}$ Dental illnesses account for more than 51 million school hours lost every year. ${ }^{7}$ The prevalence of dental caries is on the decline in developed countries and on the rise in developing countries, ${ }^{8}$ as evidenced by a prevalence of $80 \%, 64.2 \%$ and $69.2 \%$ among $2-5$ year old children in studies done in Saudi Arabia, India and Bangladesh. ${ }^{9,10}$ Similarly, a cross sectional survey in Bangladesh showed that prevalence of caries was $69.2 \%$ among $2-5$ year old, with only $44 \%$ observing correct brushing method. ${ }^{11}$

In Pakistan, according to pathfinder survey conducted in 1988, the mean DMF for all ages was 3.1. ${ }^{12}$ Independent studies conducted in Karachi, Lahore and Peshawar indicated a high prevalence of caries amongst 3-5-year-old. The highest recorded prevalence was $88.6 \%$ in Peshawar, followed by $51 \%$ in Karachi and $40.5 \%$ in a similar age bracket of children in Lahore. ${ }^{2,4,8}$ The association of dental caries with variables like age of the child, consumption of non-sweetened milk and oral hygiene was also established in this study. In Islamabad, a descriptive study conducted on 543 children in dental OPD of Pakistan Institute of Medical Sciences showed mean DMFT for each child as $6.33 .{ }^{13}$ The association of caries with low socioeconomic status, female gender and rural areas of residence too were found to be significant.

Data on the prevalence of caries among pre-school children in Pakistan is deficient. The primary dentition was the focus of our study because this age is most prone to dental caries, as evidenced by literature. This study established the base line data for prevalence of caries among preschool children of Bharakahu, Islamabad and also provided evidence on association of dental caries with certain socio-demographic characteristics. Dental caries can be prevented if factors causing it can be identified so that better preventive activities can be planned and executed. ${ }^{14}$

\section{Material and Methods}

This cross-sectional study was conducted in a peri urban area of Islamabad during the academic year of 2018. For sample size calculation, open source software called "open epi" was used. A sample size of 384 was calculated taking caries prevalence at $50 \%$ among 3-5-year-old children (as it was unknown otherwise) and 5\% margin of error, with 95\% confidence interval. A non-probability convenience sampling technique was used. Ethical consent was taken from Institutional Review Board of Islamabad Medical \& Dental College and administration of the participating schools under study. For accessibility purposes, the schools already participating in Islamabad Medical and Dental College (IM\&DC) screening program were made part of the study. The complete list of participating schools was generated and those with a preschool section identified. Once the school screening program started, the investigators accompanied the second-year dental students along with house officers to collect data. The study population included 3-5-year-old pre-school children screened during these visits. The schools that could not be reached due to unavailability of contact information or were not interested in the 
screening program were excluded from the sample. Uncooperative children or those absent on the day of data collection were also excluded. Once the estimated sample size was reached, the data was organized and analyzed.

The screening was carried out by the dental teams from IM\&DC with each team having two to three second year BDS students and one house-officer or a post graduate student for supervision. Prior to the visit, the schools were requested to set up examination stations in well-illuminated areas of the school with three chairs and a table. The Type IV examination method was used to screen children. This type of examination entails the use of a mouth-mirror and a tongue depressor only under good illumination. The child to be screened was seated across the examiner who visually examined the oral cavity with help of a tongue depressor and torch/daylight. The second team member recorded data on a standardized screening form used by the Community Dentistry Department of IM\&DC. Data including demographic information like name, age, gender along with caries prevalence (based on DMFT index), brushing habits, sugar intake and previous dental visits was recorded. The screening procedure was carried out under the supervision of a house officer or post graduate student.

The caries status was assessed using the DMFT index as defined by the WHO (1997). The DMFT index expresses the number of affected teeth in the primary dentition, with scores ranging from 0 to 20 for children. They were examined and their score expressed as the total number of decayed, missing and filled teeth in the oral cavity. The teeth that could not be unquestionably categorized as carious were not added to the score and marked sound. Furthermore, children were classified as having high DMFT (>3) and low DMFT ( $\leq 3)$ depending on the child's score. ${ }^{8}$ Socio-demographicdata such as age, gender, brushing habits, sugar consumption and previous dental visits were also recorded.
Brushing habits were categorized as brushing and no brushing, likewise sugar consumption was categorized as high and low depending on the number of high sugar content items consumed by the child. Those consuming 3 or more than three items with high sugar content were categorized as high consumers. To assess sugar consumption, teachers were asked about the lunch the children brought to class in routine, availability of sugar rich foods at the school canteen, and the preschoolers' access to the canteen. The children were also questioned about their breakfast, lunch and dinner routine. The history of previous dental visits was assessed by the presence of any restorative fillings in the mouth and also by questioning the child if he/she had ever visited a dental hospital or dentist. If the child affirmed a previous dental visit he was further questioned about the purpose of the visit for further assurance. Relevant data from the filled screening forms was extracted and analyzed for different associations using Chi square and Odd's ratio (SPSS version 22).

\section{Results}

The study sample consisted of 384 pre-school children with 220 boys and 164 girls with a mean age of $4.36 \pm 0.702$.

The prevalence of caries in preschool children of Bharakahu was $49 \%(n=189)$ with a mean DMFT score of $2.07 \pm 3.21$. Overall prevalence was higher in females (51\%) than males (48\%). Caries experience showed a progressive pattern with an increase in the DMFT score with advancing age (Table I).

The DMFT score was calculated for each child having either a decayed, missing or filled tooth and then adding them all. Regarding the dental caries status, any child having all three that is a carious lesion or a missing tooth (due to caries) or a filled tooth was accounted for in all three categories of DMFT. 


\begin{tabular}{|l|c|c|}
\hline \multicolumn{3}{|c|}{ Table I: Distribution of variables regarding dental } \\
caries status \\
\hline Variable & $\begin{array}{c}\text { DMFT } \geq 1 \\
\mathbf{n}\end{array}$ & Percentage \\
\hline Decayed (D) & 177 & 46.1 \\
\hline Missing (M) & 28 & 7.3 \\
\hline Filled (F) & 6 & 1.6 \\
\hline Gender wise distribution of DMFT \\
\hline Male (n=220) & 106 & 48 \\
\hline Female (n=164) & 83 & 51 \\
\hline Age wise distribution of DMFT \\
\hline 3yrs (n=50) & 19 & 38 \\
\hline 4yrs ( $=144)$ & 70 & 49 \\
\hline 5yrs ( $=190)$ & 100 & 53 \\
\hline
\end{tabular}

Odds ratio was used to compare the relative probabilities between variables of interest and outcomes of interest. The odds ratio showed that a low sugary diet, regular brushing and previous dental visits were significantly associated with a child caries experience and a low DMFT score. The most significant association was for brushing and low DMFT. Children who brushed their teeth regularly (at least once a day) were 4 times more likely to have a low DMFT score of $<1$ than those who did not brush at all $(P<0.001)$. Likewise, those who had visited a dentist in the past were twice more likely to have a low $\operatorname{DMFT}(P=0.003)$. The association of low sugar consumption and a low Children with carious lesions and a DMFT score of $>1$ were $46 \%(n=177)$. Out of these $55 \%(n=97)$ had a low DMFT score of $\leq 3$ whereas $45 \%(n=80)$ had a high DMFT score of $>3$ teeth (Table II).

\section{Table II: Severity of decayed component (D) of DMFT}

\begin{tabular}{|l|c|c|}
\hline Variable & Frequency & Percentage \\
\hline Dental Caries Status (D) (n= 384) \\
\hline Positive & 177 & 46.1 \\
\hline Negative & 207 & 53.9 \\
\hline Decayed category for severity (D) (n=177) \\
\hline 1-3 (low) teeth & 97 & 55 \\
\hline 4+ (high) teeth & 80 & 45 \\
\hline
\end{tabular}

In the children with a DMFT score of $\geq 1$, the decayed component accounted for $94 \%$ of the score. In the two categories of low and high DMFT score, $54.5 \%(n=103)$ children had a low DMFT score and $45.5 \%(n=86)$ had a high DMFT score of 3 or above. ${ }^{8}$ Median DMFT score was found to be 3 .

Regarding oral hygiene practices of pre-school children, $90.6 \%(n=348)$ cleaned their teeth at least once a day and only $9.4 \%(n=36)$ confessed to no brushing at all. The percentage of children on high sugary diet was greater at $58.9 \%(n=226)$ than children on a low sugar diet. Majority of the study population (81.8\%; $n=314$ ) had never visited a dentist or a dental hospital previously.

DMFT score was statistically insignificant (OR 1.4; $P=0.878$ ). Hence, brushing turned out to be the most significant factor in determining the caries experience for a child (Table III).

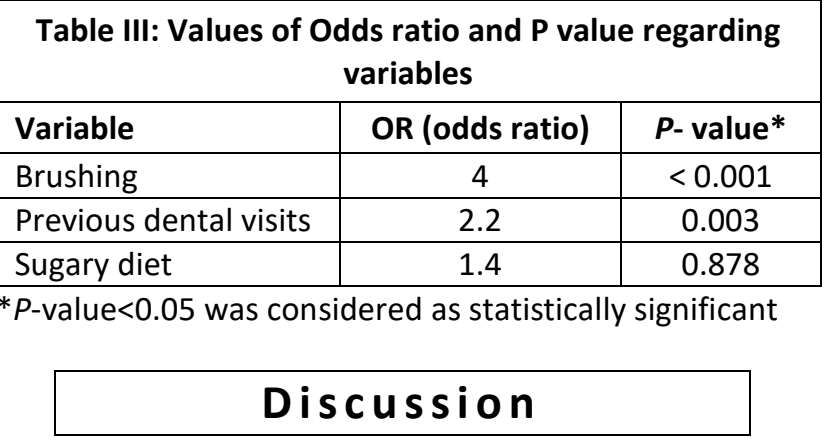

According to $\mathrm{WHO}$, caries is the most prevalent non communicable disease globally. In 2015, the "Global Burden of Disease Study" established its high prevalence ranking it "first" for decay of permanent teeth (2.3 billion people) and 12th for deciduous teeth (560 million children). ${ }^{15}$ It affects humans of all ages in all parts of the world, because it involves an inter-play of social, behavioral, cultural, dietary and biological risks that are associated with its initiation and progress. ${ }^{16}$ However, caries can easily be prevented through simple and cost-effective interventions, especially in countries where treatment is unaffordable or inaccessible. 
Globally, the statistics for caries prevalence in children of developing nations is quite distressing. A systematic review conducted in Saudi Arabia showed that $80 \%$ of the children with primary dentition had caries. ${ }^{9}$ The prevalence of $64.2 \%$, $67.9 \%$ 69.2\% and $95.4 \%$, was reported in studies conducted in India, Argentine, Bangladesh and Thailand, respectively. ${ }^{10,11,14,17}$ Conversely, preschool children in industrialized nations have lower caries prevalence with $34 \%$ in Great Britain, ${ }^{18}$ $40 \%$ in Singapore, ${ }^{19} 20 \%$ (among 3 year old) and $52 \%$ (among 5 year old) respectively in Norway. ${ }^{20}$

The present study reported a caries prevalence of $49 \%$ in preschool children. This percentage is quite high in perspective of biological consequences and financial burden of treating the disease in a low budget healthcare system. Two Indian studies showed prevalence of $53 \%$ and $54 \%$ among $3-5$ year olds. ${ }^{21,22}$ The similarity in distribution was perhaps due to similarities in culture, dietary patterns and socio demographic characteristics. However, there is a great disparity in caries prevalence in preschoolers among different cities of Pakistan with $40.5 \%$ in Lahore, $51 \%$ in Karachi, $88.6 \%$ in Peshawar and $90 \%$ in Hyderabad. ${ }^{2-4,8}$

In our study, decayed teeth formed the major portion (94\%) of total DMFT score. This is a common finding in most of the literature, e.g. $90 \%$ in a Bangladeshi study and $69 \%$ in a Singaporean study. ${ }^{11,19}$ The reasons for children's under treatment can be multifactorial ranging from dental anxiety, treatment cost, lack of parent's awareness, to the dearth of affordable and accessible dental services in low and middle income countries. This fact is supported by the finding that the number of children who had visited a dental hospital/ clinic is much more than the number of fillings observed in our study sample. The possible explanation can be that in case of pain, they go to the hospital, take medication and even go for extraction to get rid of pain, but they do not pursue the treatment in the form of fillings or other restorative procedures.
Previous dental visits are also found to be low in studies of other developing nations, like India and Argentine. ${ }^{14,21}$ Regarding dental visits, another point that can be deduced is that those who go to the dentist stay more aware about oral hygiene, and therefore have low caries and ultimately less fillings.

The female children in our study had higher caries prevalence (51\%) as compared to males (48\%). A Pakistani study also showed higher caries prevalence in females (58.4\%), ${ }^{2}$ whereas, a Lahorebased study showed a prevalence of $54 \%$ in the male population. ${ }^{8}$ Regarding the disease severity among caries positive children in our study, $54 \%$ had 1-3 teeth involved whereas, $46 \%$ had 4 or more teeth involved. It is very rare to find children with greater number of teeth involved in DMFT in the literature as well. For example, $54 \%$ of the study population has 1-3 teeth involved in a study conducted on preschool children in Hyderabad and $45 \%$ have more than 3 teeth involved. ${ }^{3}$ The decay of one or two teeth can make children more conscious of their oral health status and prompt them to adapt better oral hygiene practices, thus restricting further decay of teeth.

The DMFT score of 3-5 years old children incremented with age i.e. from $38 \%$ among 3-yearold to $49 \%$ and $53 \%$ among 4 and 5-year-old, respectively. Similar findings have been reported in literature. For example, caries prevalence increasing from $33 \%$ to $48 \%$ to $75 \%$ among 3,4 and 5 years of age respectively in a Pakistani study and increasing from $26 \%$ to $37 \%$ to $49 \%$ in a Singaporean study. ${ }^{8,19}$ The most convincing rationale of this caries advancement would be the shift of dietary habits from healthy homemade food to unhealthy snacks available at canteen, decreased involvement of parents in tooth brushing with advancing age and the increased duration of teeth exposure to oral environment. ${ }^{11}$ 
The strongest association of DMFT observed in our study was with brushing followed by its association with previous dental visits. No statistically significant association found with sugar consumption in our study can be attributed to the less reliable information collected. As the children were 3-5-year-old and they could give wrong information regarding the frequency of sugar consumption. However, considering them all together, they all contribute in caries etiology. For example, some children consume high amount of sugars but have low DMFT, because they take care of the oral hygiene very well. On the other hand, some children clean their teeth regularly but are found with high DMFT scores, because they consume large amounts of sugar. All this reaffirms the significance of brushing, dental visits and sugar consumption in maintaining a healthy oral status in children.

\section{Conclusion}

This study provided us with the baseline data regarding the prevalence of caries in primary school going children of Bharakahu, Islamabad. The study also accentuated the significance of brushing in these young children. The prevalence of unmet dental treatment needs was also highlighted with a high number of 'decayed teeth' in these children. Raising awareness and training these youngsters is the key to a better oral health status. One can certainly address these issues through the endowment of oral health education or promotion programs in their schools. Ideally teachers are the best guides to steer these young children in the right direction. The primary school teachers should undertake the role of mentors and train these children to adopt healthy oral hygiene practices. Our focus should switch to these youngsters so that our future generations enjoy good oral health.

Limitations: Since it was a cross sectional study, it could not establish any temporal association.
Another limitation of the study was the complete reliability of the information collected as the study population was very young i.e. 3-5-year-old preschool children.

Future recommendations: More research needs to be conducted on communities in the rural and peri-urban areas of Islamabad, Pakistan so that reported data may be consolidated. The key to preventing caries in these communities is raising awareness regarding the importance of oral health. Children are the ideal audiences for such oral health promotional activities. Thus, schools in these areas should collaborate with dental institutes and integrate oral health awareness programs in their curricula.

\section{Acknowledgement}

We are really thankful to the dental students and house officers, who helped in data collection. We specially acknowledge the administration of the schools for giving permission to conduct the screening. A special word of thanks to all the 3-5 years old children who were part of this project.

\section{References}

1. Bader JD, Rozier RG, Lohr KN, Frame PS. Physicians' roles in preventing dental caries in preschool children: a summary of the evidence for the U.S. Preventive Services Task Force. AJPM. 2004; 26(4): 315-25. Doi: 10.1016/j.amepre.2003.12.001

2. Sufia $S$, Chaudhry S, Izhar F, Syed A, Qayum Mirza BA, Ali Khan A. Dental caries experience in preschool children- Is it related to a child's place of residence and family income? Oral Health Prev Dent. 2011; 9(4): 375-9.

3. Sahito N, Sahito MA, Fazlani KA. Prevalence of caries among school children in Hyderabad Pakistan. IJAS. 2015; 2(1):034-8.

4. Ahmad S, Khan H, Khan M, Maryam W. Prevalence and patterns of early childhood caries among school children in Peshawar. JKCD. 2015; 6(1): 36-9. 
5. Rafael da Silveira Moreira (2012). Epidemiology of Dental Caries in the World, Oral Health Care Pediatric, Research, Epidemiology and Clinical Practices, Prof. Mandeep Virdi (Ed.), ISBN: 978-95351-0133-8, In Tech, Available from: http://www.intechopen.com/books/oral-healthcare-pediatric-research-epidemiology-andclinicalpractices/epidemiology-of-dental-caries-in-theworldhttp://cdn.intechopen.com/pdfs/29340/InTec h-Epidemiology_of_dental_caries_in_the_world.pdf

6. Baghdady VS, Ghose LJ. Dental caries prevalence in schoolchildren of Baghdad province, Iraq. Community Dent Oral Epidemiol. 1982; 10(3): 14851. Doi: 10.1111/j.1600-0528.1982.tb01340.x

7. U.S department of health and human services (USDHHS). Oral health in America: a report of surgeon general. National institute of dental and craniofacial research. 2000.

8. Dawani N, Nisar N, Khan N, Syed S, Tanweer N. Prevalence and factors related to dental caries among pre-school children of Saddar town, Karachi, Pakistan: a cross-sectional study. BMC Oral Health. 2012; 12(1): 59. Doi: 10.1186/1472-6831-12-59

9. Al Agili DE. A systematic review of population-based dental caries studies among children in Saudi Arabia. Saudi dent J. 2013; 25(1): 3-11. Doi: 10.1016/j.sdentj.2012.10.002

10. Reddy KS, Reddy S, Ravindhar P, Balaji K, Reddy H, Reddy A. Prevalence of dental caries among 6-12 years school children of Mahboobnagar district, Telangana state, India: A cross - sectional study. Indian J Dent Sci. 2017; 9 (1): 1-7

11. Khan MAI, Raihan MM, Hossain MM, Shahin AS. Dental Caries and associated factors among 2-5 years children attending OPD of a selected dental hospital in Bangladesh. Update Dent Coll J. 2011; 1(1): 2-5. Doi: 10.3329/updcj.v1i1.13945

12. Mehar R. Dental disorders in Pakistan - A national pathfinder survey. JPMA. 1991; 41(10): 250-2.

13. Abdullah S, Qazi H S, Maxood A. Dental caries status in 6-9 years old children. PODJ; 2008; 28(1): 107-12

14. Llompart G, Marin GH, Silberman M, Merlo I, Zurriaga O. Oral health in 6-year old schoolchildren from berisso, Argentina: falling far short of WHO goals. Med Oral Patol Oral Cir Bucal. 2010;15(1):101-5.

15. Sugars and dental caries technical information note. https://www.who.int/oral_health. [cited 2019 Dec 25], [updated 2017]; available from https://www.who.int/oral_health/publications/ sugars-dental-caries-keyfacts/en/.

16. Albino J, Tiwari T. Preventing childhood caries a review of recent behavioral research. J Dent Res. 2016; 95(1): 35-42

17. Sutthavong S, Taebanpakul S, Kuruchitkosol C, Ayudhya TI, Chantveerawong T, Fuangroong $\mathrm{S}$, et al. Oral health status, dental caries risk factors of the children of public kindergarten and schools in Phranakornsriayudhya, Thailand. J Med Assoc Thai. 2010; 93(Suppl 6): S71-S78.

18. Pitts NB, Boyles J, Nugent ZJ, Thomas N, Pine CM. The dental caries experience of 5-year old children in Great Britain (2005/6). Surveys Co-ordinated by the British association for the study of community dentistry. Community Dent Health. 2007; 24(1): 5963. PMID: 17405473

19. Gao XL, Hsu CYS, Loh T, Koh D, Hwang HB, Xu Y. Dental caries prevalence and distribution among preschoolers in Singapore. Community Dent Health. 2009; 26(1): 12-7.

20. Skeie MS, Edpelid I, Skaare AD, Gimmestad A. Caries patters in an urban preschool population in Norway. Europ J of Paed Dent. 2005; 6(1): 16-22.

21. Simratvir $M$, Moghe GA, Thomas AM, Singh N, Chopra S. Evaluation of caries experience in 3-6year-old children, and dental attitudes amongst the care-givers in the Ludhiana city. J Indian Pedod Prev Dent. 2009; 27(3): 164-9.

22. Mahejabeen R, Sudha P, Kulkarni SS, Anegundi R. Dental caries prevalence among preschool children of Hubli, Dharwad city. J Indian Soc Pedod Prev Dent. 2006; 24(1): 19-22. 